\title{
MULTIPLIERS FOR AMALGAMS AND THE ALGEBRA $S_{0}(G)$
}

MARIA L. TORRES DE SQUIRE

1. Notation and background material. Throughout the whole paper $G$ will be a locally compact abelian group with Haar measure $m$ and dual group $\hat{G}$. The difference of two sets $A$ and $B$ will be denoted by $A \sim B$, i.e.,

$$
\begin{aligned}
& A \sim B=\{x \in A \mid x \notin B\} \text { and } \\
& A-B=\{x \in y \mid x \in A \text { and } y \in B\} .
\end{aligned}
$$

For a function $f$ on $G$ and $s \in G$, the functions $f^{\prime}$ and $f_{s}$ will be defined by

$$
f^{\prime}(x)=f(-x) \text { and } f_{s}(x)=f(x-s)(x \in G) .
$$

As usual $C_{0}(G)=C_{0}$, and $C_{c}(G)=C_{c}$, will be the linear space of continuous functions on $G$ which vanish at infinity, and have compact support, respectively. For $E \subset G$ compact, $C_{E}(G)=C_{E}$ will denote the space of functions $f \in C_{c}(G)$ whose support is included in $E$, i.e., $\operatorname{supp} f \subset E$, endowed with the supremum norm; $D_{E}(G)$ will be the Banach space of functions

$$
f=\sum g_{i}{ }^{*} h_{i}
$$

where $g_{i}, h_{i}$ are in $C_{E}(G)$ and

$$
\|f\|_{E}=\sum\left\|g_{i}\right\|_{\infty}\left\|h_{i}\right\|_{\infty}<\infty
$$

defined in [12, Section 2], and $D(G)$ will denote the internal inductive limit of the spaces $D_{E}(G)$. That is, $D(G)=\cup D_{E}(G)$ and the neighborhood bases of the origin are of the form

$$
U_{\epsilon}=\left\{f \mid f \in D_{E}(G),\|f\|_{E}<\epsilon\right\} .
$$

A quasimeasure is an element of the continuous dual $Q(G)$ of $D(G)$. We will note by $L_{\text {loc }}^{p}(1 \leqq p \leqq \infty)$ the space of measurable functions $f$ on $G$ such that $f$ restricted to any compact subset $E$ of $G$ belongs to $L^{p}(G)$, i.e., $f \mid E \in L^{p}$. The space of Radon measures on $G$ will be denoted by $V(G)$. The pairing between a Banach space $B$ and its dual $B^{*}$ will be denoted by $\langle$,$\rangle . That is,$

Received May 21, 1985 and in revised form November 25, 1985. The author wishes to thank Dr. J. Stewart for financial support during the preparation of this paper. This paper is dedicated to Professor Angel Carrillo. 


$$
\langle h, \sigma\rangle=\sigma(h) \quad\left(h \in B, \sigma \in B^{*}\right) .
$$

For $\hat{x} \in \hat{G}$, we put

$$
[x, \hat{x}]=\hat{x}(x) \quad(x \in G),
$$

hence the Fourier-Stietljes (inverse Fourier-Stietljes) transform of a bounded measure $\mu$ on $G$ (on $\hat{G}$ ) will be a function $\hat{\mu}(\stackrel{v}{\mu})$ on $\hat{G}$ (on $G$ ) defined by

$$
\begin{aligned}
& \hat{\mu}(\hat{x})=\int_{G} \overline{[x, \hat{x}]} d \mu(x)=\int_{G}[-x, \hat{x}] d \mu(x) \\
& \left(\stackrel{v}{\mu}(x)=\int_{\hat{G}}[x, \hat{x}] d \mu(\hat{x})\right) .
\end{aligned}
$$

For a bounded measure $\mu$ on $G$ (on $\hat{G}$ ) we define

$$
\begin{aligned}
& \stackrel{\vee}{\mu}(\hat{x})=\hat{\mu}^{\prime}(\hat{x})=\hat{\mu}(-\hat{x}) \\
& \left(\hat{\mu}(x)=\breve{\mu}^{\prime}(x)=\stackrel{\vee}{\mu}(-x)\right) .
\end{aligned}
$$

The following definition of amalgam spaces and spaces of unbounded measures of type $q$ is due to J. Stewart [21]. For a definition of these spaces on locally compact not necessarily abelian groups see [1] or [5].

Definition 1.1. By the Structure Theorem [15, Theorem 24.30] $G$ is topologically isomorphic to $\mathbf{R}^{a} \times G_{1}$, where $a$ is a nonnegative integer and $G_{1}$ is a locally compact abelian group which contains an open compact subgroup $H$. Let

$$
L=[0,1)^{a} \times H \text { and } J=\mathbf{Z}^{a} \times T,
$$

where $T$ is a transversal of $H$ in $G_{1}$, i.e.,

$$
G_{1}=\cup\{t+H \mid t \in T\} .
$$

For $\alpha \in J$ we define $L_{\alpha}=\alpha+L$, and therefore $G$ is equal to the disjoint union of relatively compact sets $L_{\alpha}$. We normalize $m$ so that

$$
m(L)=m\left(L_{\alpha}\right)=1 \text { for all } \alpha .
$$

Let $1 \leqq p, q \leqq \infty$. The amalgam space

$$
\left(L^{p}, l^{q}\right)(G)=\left(L^{p}, l^{q}\right)
$$

is the linear space

$$
\left\{f \in L_{\mathrm{loc}}^{p} \mid\|f\|_{p q}=\left[\sum_{\alpha}\left[\int_{L_{\alpha}}|f|^{p}\right]^{q / p}\right]^{1 / q}<\infty\right\}
$$

endowed with the norm $\|\cdot\|_{p q}$ and the space $M_{q}(G)=M_{q}$ of unbounded measures of type $q$ is the linear space 


$$
\left\{\mu \in V(G) \mid\|\mu\|_{q}=\left[\sum_{\alpha}|\mu|\left(L_{\alpha}\right)^{q}\right]^{1 / q}<\infty\right\}
$$

endowed with the norm $\|\cdot\|_{q}$; with the appropriate changes when $p, q$ are infinite.

Definition 1.2. For $1 \leqq p, q \leqq \infty$ we define the amalgams:

i) $\left(C_{0}, l^{q}\right)=\left(C_{0}, l^{q}\right)(G)=C_{0}(G) \cap\left(L^{\infty}, l^{q}\right)(G)$.

ii) $\left(L^{p}, c_{0}\right)=\left(L^{p}, c_{0}\right)(G)$ is the space of functions in $\left(L^{p}, l^{\infty}\right)$ such that

$$
\lim \|f\|_{L^{p}\left(L_{\alpha}\right)}=0 .
$$

That is, given $\epsilon>0$ there exists $F \subset J$ (finite) such that

$$
\|f\|_{L^{p}\left(L_{\alpha}\right)}<\epsilon \text { for all } \alpha \notin F \text {. }
$$

The Banach spaces $\left(L^{p}, l^{q}\right)$ and $M_{q}(1 \leqq p, q \leqq \infty)$ satisfy the following inclusion relations and inequalities [21, p. 1284].

$$
\begin{array}{lll}
\text { (1.1) } & \left(L^{p}, l^{q_{1}}\right) \subset\left(L^{p}, l^{q_{2}}\right) & q_{1} \leqq q_{2} \\
(1.2) & \left(L^{p_{1}}, l^{q}\right) \subset\left(L^{p_{2}}, l^{q}\right) & p_{1} \geqq p_{2} \\
(1.3) & \left(L^{p}, l^{q}\right) \subset L^{p} \cap L^{q} \quad p \geqq q \\
\text { (1.4) } & \left(L^{p}, l^{p}\right)=L^{p} & \\
\text { (1.5) } \quad M_{q} \subset M_{s} & q \leqq s \\
\text { (1.6) }\|f\|_{p q_{2}} \leqq\|f\|_{p q_{1}} & q_{2} \geqq q_{1} \\
\text { (1.7) }\|f\|_{p_{2} q} \leqq\|f\|_{p_{1} q} & p_{1} \geqq p_{2} \\
\text { (1.8) }\|\mu\|_{s} \leqq\|\mu\|_{q} & q \leqq s .
\end{array}
$$$$
\text { (1.5) } M_{q} \subset M_{s}
$$

Note that the usual $L^{p}$ spaces are particular cases of amalgams and that $C_{c}$ and $\left(C_{0}, l^{1}\right)$ are included in all amalgam spaces.

If $f \in\left(L^{1}, l^{q}\right)(1 \leqq q \leqq \infty)$ then the measure $f m$ (where $\int g d f m$ $=\int g f d m$ ) belongs to $M_{q}$ and

$$
\|f m\|_{q}=\|f\|_{1 q} .
$$

Hence $f \mapsto f m$ is a natural (isometric) embedding from $\left(L^{1}, l^{q}\right)$ into $M_{q}$. In this sense we say that $\left(L^{1}, l^{q}\right) \subset M_{q}$. Therefore from (1.2) and (1.5)

$$
\left(L^{p}, l^{q}\right) \subset\left(L^{1}, l^{q}\right) \subset M_{q} \subset M_{\infty} \text { for } 1 \leqq p, q \leqq \infty .
$$

Remark 1.3. Since $\hat{G}=\mathbf{R}^{a} \times \hat{G}_{1}$ and $\hat{G}_{1}$ contains the open compact subgroup $\mathscr{H}$ which is the annhilator of $H,(\mathscr{H}=\{x \in G \mid[x, \hat{x}]=1$ for all $x \in H\}$ ) we can choose $\mathscr{H}$ to define

$$
\hat{L}=[0,1)^{a} \times \mathscr{H}
$$


and for $\beta \in I, I=\mathbf{Z}^{a} \times T^{\prime}$ where

$$
\hat{G}_{1}=\cup\left\{t+\mathscr{H} \mid t \in T^{\prime}\right\}, \quad L_{\beta}=\beta+\hat{L} .
$$

Then using $\left\{L_{\beta}\right\}_{I}$ we can define as in Definition 1.1 the amalgam spaces $\left(L^{p}, l^{q}\right)(\hat{G})$ and the spaces of unbounded measures $M_{q}(\hat{G})$, [21, Section 3].

Hereafter $a, G_{1}, H, \mathscr{H}, J, I, L$ and $\hat{L}$ will be as in Definition 1.1 and Remark 1.3.

We will state now the results we will need in the next sections.

TheOREM 1.4 [21, Theorems 3.2 and 4.3], [22, Theorems 3.1 and 3.2].

i) Let $1 \leqq p, q<\infty$. The amalgam $\left(L^{p^{\prime}}, l^{q^{\prime}}\right)\left(\left(L^{p^{\prime}}, l^{1}\right)\right)$ is isometrically isomorphic to $\left(L^{p}, l^{q}\right)^{*}\left(\left(L^{p}, c_{0}\right)^{*}\right)$ via the map $g \mapsto\langle f, g\rangle$, where

$$
\langle f, g\rangle=\int_{G} f g d x
$$

$\left(g \in\left(L^{p^{\prime}}, l^{q^{\prime}}\right)\left(\left(L^{p^{\prime}}, l^{1}\right)\right), f \in\left(L^{p}, l^{q}\right)\left(\left(L^{p}, c_{0}\right)\right)\left(p^{\prime}\right.\right.$ being the conjugate of $p)$.

\section{Moreover}

$$
\begin{aligned}
& |\langle f, g\rangle| \leqq\|f\|_{p q}\|g\|_{p^{\prime} q^{\prime}} \\
& |\langle f, g\rangle| \leqq\|f\|_{p \infty}\|g\|_{p^{\prime} 1} .
\end{aligned} \quad \text { (Hölder inequality) }
$$

ii) Let $1 \leqq q \leqq \infty$. If $T \in\left(C_{0}, l^{q}\right)^{*}$ then there exists a unique $\mu \in M_{q^{\prime}}$ such that

$$
T(f)=\int_{G} f d \mu \quad\left(f \in\left(C_{0}, l^{q}\right)\right)
$$

and

$$
\begin{array}{ll}
\|T\| \leqq\|\mu\|_{q^{\prime}} \leqq 2^{a}\|T\| & \text { if } 1 \leqq q<\infty \\
\|T\|=\|\mu\|_{1} & \text { if } q=\infty .
\end{array}
$$

Hence

$$
|\langle f, g\rangle|=\left|\int f g\right| \leqq\|f\|_{\infty q}\|g\|_{1 q^{\prime}}\left(f \in\left(C_{0}, l^{q}\right), g \in\left(L^{1}, l^{q^{\prime}}\right)\right) .
$$

TheOREM 1.5 [22, Theorem 3.14]. Let $1 \leqq p, q<\infty, 1 \leqq s \leqq \infty$. If $f$ belongs to any of the amalgams $\left(L^{p}, l^{q}\right),\left(L^{p}, c_{0}\right),\left(C_{0}, l^{s}\right)$, then the map $s \mapsto f_{s}$ is continuous on $G$.

Theorem 1.6 [2, Section 7, i) ], [5, Theorem 4.2], [22, Theorems 4.7 and 4.8]. If $p, q, r, s$ are exponents such that

$$
\begin{aligned}
& 1 / p+1 / r-1=1 / m \leqq 1 \text { and } \\
& 1 / q+1 / s-1=1 / n \leqq 1
\end{aligned}
$$

then 
a) $\left(L^{p}, l^{q}\right) *\left(L^{r}, l^{s}\right) \subset\left(L^{m}, l^{n}\right)$

b) $\left(L^{p}, l^{q}\right) *\left(L^{p^{\prime}}, l^{q^{\prime}}\right) \subset C_{0} \quad 1 \leqq p \leqq \infty, 1<q<\infty$

c) $\left(L^{p}, l^{q}\right) *\left(L^{p^{\prime}}, l^{s}\right) \subset\left(C_{0}, l^{n}\right) \quad 1 \leqq p, s \leqq \infty, 1<q<\infty$

d) $\left(L^{p}, l^{q}\right) *\left(L^{r}, l^{q^{\prime}}\right) \subset\left(L^{m}, c_{0}\right) \quad 1 \leqq p, r \leqq \infty, 1<q<\infty$

e) $\left(L^{p}, c_{0}\right) *\left(L^{p^{\prime}}, l^{1}\right) \subset C_{0} \quad 1<p<\infty$

f) $\left(L^{1}, c_{0}\right) *\left(L^{\infty}, l^{1}\right) \subset\left(L^{\infty}, c_{0}\right)$

g) $\left(L^{p}, l^{q}\right) * M_{s} \subset\left(L^{p}, l^{n}\right) \quad 1 \leqq p, q, s \leqq \infty$

h) $\left(L^{p}, l^{q}\right) * M_{q^{\prime}} \subset\left(L^{p}, c_{0}\right) \quad 1 \leqq p \leqq \infty, 1<q<\infty$

i) $\left(C_{0}, l^{q}\right) * M_{s} \subset\left(C_{0}, l^{n}\right) \quad 1 \leqq q \leqq \infty, 1 \leqq s<\infty$

j) $\left(L^{\infty}, l^{q}\right) * M_{q^{\prime}} \subset\left(L^{\infty}, c_{0}\right) \quad 1<q<\infty$.

Moreover if $f \in\left(L^{p}, l^{q}\right), g \in\left(L^{r}, l^{s}\right), \mu \in M_{s}$, then Young's inequalities for amalgams are:

$$
\begin{aligned}
& \|f * g\|_{m n} \leqq 2^{a}\|f\|_{p q}\|g\|_{r s} \quad \text { if } m \neq 1 \\
& \|f * g\|_{1 n} \leqq 2^{2 a}\|f\|_{1 q}\|g\|_{1 s} \\
& \|f * \mu\|_{p n} \leqq 2^{a}\|f\|_{p q}\|\mu\|_{s} \quad \text { if } p \neq 1 \\
& \|f * \mu\|_{1 n} \leqq 2^{2 a}\|f\|_{1 q}\|\mu\|_{s} .
\end{aligned}
$$

It follows from Theorem 1.6 that all amalgams and all $M_{q}$ spaces are $M_{1^{-}}$and $L^{1}$-modules [7, Definition 14.1] and that the spaces $\left(L^{p}, l^{1}\right)$ $(1 \leqq p \leqq \infty),\left(C_{0}, l^{1}\right)$ and $M_{1}$ are algebras under convolution.

Definition 1.7. A net $\left\{e_{n}\right\}$ in a commutative, normed algebra $A$ is an approximate identity, abbreviated a.i., if for all $a \in A, \lim a e_{n}=a$ in $A$.

Proposition 1.8. [22, Corollary 4.14]. Let $A$ be any of the amalgams $\left(L^{p}, l^{q}\right),\left(L^{p}, c_{0}\right),\left(C_{0}, l^{s}\right)(1 \leqq p, q<\infty, 1 \leqq s \leqq \infty)$. If $\left\{e_{n}\right\}$ is an a.i. in $L^{1}$, then

$$
\lim \left\|e_{n} * f-f\right\|_{A}=0 \quad \text { for all } f \in A .
$$

2. The algebra $S_{0}(G)$. The algebra $S_{0}(G)$ was originally defined by H. G. Feichtinger [10] and studied independently by J. P. Bertrandias [3]. We will denote by $A(G)$ the Fourier algebra of functions $f$ in $C_{0}(G)$ such

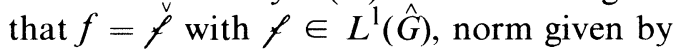

$$
\|f\|_{A}=\|f\|_{1}
$$

and pointwise multiplication. The space $A_{c}(G)$ will be the intersection of $A(G)$ and $C_{c}(G)$, and for a compact subset $E$ of $G$, we define

$$
A_{E}(G)=\left\{f \in A_{c}(G) \mid \operatorname{supp} f \subset E\right\} .
$$

The definition of $S_{0}(G)$ is based on a bounded uniform partition of 
unity in $A(G)$. We will give an explicit construction of such a partition for the sake of completeness.

Definition 2.1. Consider the following function $f: \mathbf{R} \rightarrow \mathbf{R}$ given by

$$
f(x)= \begin{cases}0 & \text { if }|x| \geqq 1 \\ 1-|x| & \text { if }|x| \leqq 1\end{cases}
$$

Since

$$
\hat{f}(x)=2 / \sqrt{2 \pi}\left(1-\cos x / x^{2}\right) \text { and } \operatorname{supp} f=[-1,1],
$$

we conclude that $f \in A_{c}(\mathbf{R})$. For $n \in \mathbf{Z}$ we define $f_{n}$ to be the function $f_{n}(x)=f(x-n)$ on $R$. It is clear that

$$
f_{n} \in A_{c}(\mathbf{R}) \text { and } \operatorname{supp} f_{n}=n+\operatorname{supp} f=[n-1, n+1] .
$$

Moreover for each $x \in \mathbf{R}$,

$$
\sum_{n} f_{n}(x)=1
$$

For $s=(x, t)=\left(x_{1}, \ldots, x_{a}, t\right)$ in $G=R^{a} \times G_{1}$ we define the function $\psi: G \rightarrow \mathbf{R}$ by

$$
\psi(x, t)=f\left(x_{1}\right) \ldots f\left(x_{a}\right) \cdot \chi_{H}(t)
$$

since $f$ and $\chi_{H}$ belong to $A_{c}$ ( $H$ is compact and $\hat{\chi}_{H}=\chi_{\mathscr{H}}$ ) we have that

$$
\psi \in A_{c}(G) \text { and supp } \psi=[-1,1]^{a} \times H .
$$

Then for $\alpha=\left(m_{1}, \ldots, M_{a}, t\right)$ in $J$ the function

$$
\psi_{\alpha}=f_{m_{1}} \ldots f_{m_{a}} \cdot \chi_{t+H}
$$

has the following properties:

P.1) $\psi_{\alpha} \in A_{c}(G)$

P.2) $\operatorname{supp} \psi_{\alpha}=\alpha+\operatorname{supp} \psi$

P.3) $\quad \sum \psi_{\alpha}(s)=1$ for all $s \in G$

P.4) $\sup \left\|\psi_{\alpha}\right\|_{A} \leqq\|\psi\|_{A}$.

Therefore $\left\{\psi_{\alpha}\right\}_{J}$ is a bounded uniform partition of unity in $A(G)[\mathbf{1 1}$, Definition 2].

Definition 2.2. Let $\left\{\psi_{\alpha}\right\}_{J}$ be the family defined above. Then $S_{0}(G)=S_{0}$ is the linear space of continuous functions $f$ in $A(G)$ such that

$$
\|f\|_{S_{0}}=\Sigma\left\|f \psi_{\alpha}\right\|_{A}<\infty
$$

endowed with the norm $\|\cdot\|_{S_{0}}$.

It follows from [11, Theorem 2] that Definition 2.2 is equivalent to Feichtinger's original definition of $S_{0}$ in [10], [11] and that it is 
independent of the partition of unity chosen. The following are some of the properties of $S_{0}(G)$. For a proof see [11] and [19].

P.5) $S_{0}(G)$ is a Segal algebra. Hence it is an $L^{1}$-module and has an a.i. $\left\{e_{n}\right\}$ such that $\left\|e_{n}\right\|_{1}=1$ for all $n$ [20, Section 8, Proposition 1 ii) ].

P.6) $A_{c}(G)$ is dense in $S_{0}(G)$.

P.7) $M_{\infty} \subset S_{0}(G)^{*} \subset Q(G)$.

P.8) $S_{0}(G) \subset\left\{f \in\left(C_{0}, l^{\mathrm{l}}\right)(G) \mid \hat{f} \in\left(C_{0}, l^{1}\right)(\hat{G})\right\}$.

P.9) $S_{0}(G)^{\wedge}=S_{0}(\hat{G})$.

Definition 2.3. [10, Theorem B2]. The Fourier transform $\hat{\sigma}$ of $\sigma \in S_{0}(G)^{*}$ is an element of $S_{0}(\hat{G})^{*}$ given by

$$
\langle h, \hat{\sigma}\rangle=\left\langle\breve{h}^{\prime}, \sigma\right\rangle=\langle\hat{h}, \sigma\rangle \quad\left(h \in S_{0}(\hat{G})\right) .
$$

Similarly the inverse Fourier transform $\stackrel{\vee}{\sigma}$ of $\sigma \in S_{0}(\hat{G})^{*}$ is an element of $S_{0}(G)^{*}$ given by

$$
\langle h, \stackrel{\vee}{\sigma}\rangle=\left\langle\hat{h}^{\prime}, \sigma\right\rangle=\langle\grave{h}, \sigma\rangle \quad\left(h \in S_{0}(G)\right) .
$$

It is clear from P.9) that $\hat{\sigma}$ and $\stackrel{v}{v}$ are well defined and by P.7) and (1.9) Definition 2.3 provides a definition for a Fourier transform on all amalgam spaces and all spaces of unbounded measures of type $q$.

Remark 2.4. i) By P.8) any $h$ in $S_{0}(G)$ is equal to the inverse of its Fourier transform, i.e., $h=(\hat{h})^{\vee}$. Hence for any $\psi_{\alpha}$ (as in Definition 2.1)

$$
\left\|h \psi_{\alpha}\right\|_{A}=\left\|\hat{h} * \hat{\psi}_{\alpha}\right\|_{1}
$$

and,

$$
\left.\left\{h \psi_{\alpha}\right\}_{J} \subset\left(C_{0}, l^{1}\right) \text { by }[2, \text { Section } 7 \mathrm{~h})\right] .
$$

ii) If $\sigma \in S_{0}(G)^{*}$, then $\sigma=(\hat{\sigma})^{\vee}$ by i).

iii) From Definition 2.3 it follows immediately that if $\sigma, \eta \in S_{0}(G)^{*}$ and $\hat{\sigma}=\hat{\eta}$, then $\sigma=\eta$.

Proposition 2.5. Let $A$ be as in Proposition 1.8. Then $S_{0}(G)$ is dense in $A$.

Proof. It is enough to prove that $D(G)$ is dense in $\left(C_{c},\|\cdot\|_{A}\right)$ because $A_{c}(G)$ is dense in $S_{0}(G), A_{c}(G)$ and $D(G)$ are homeomorphically isomorphic as spaces of functions on $G\left[6\right.$, Theorem 3.1] and $C_{c}(G)$ is dense in $A$ [2, Section 7, e) ].

Let $\phi \in C_{c}(G)$ with supp $\phi=E$, and let $\left\{e_{n}\right\}$ be an a.i. in $L^{1}(G)$ such that $\left\{e_{n}\right\} \subset C_{k}(G)$ for some fixed $K \subset G$. Hence $\left\{\phi * e_{n}\right\} \subset D(G)$ and, by Proposition 1.8,

$$
\lim \left\|\phi * e_{n}-\phi\right\|_{A}=0 .
$$

Proposition 2.5 together with Theorem 1.4 gives a necessary and sufficient condition for an element of $S_{0}(G)^{*}$ to be in an amalgam or $M_{q}$ space. 
Proposition 2.6. Let $\sigma \in S_{0}(G)^{*}$. Then $\sigma$ belongs to $\left(L^{p}, l^{q}\right)(1<p \leqq \infty$, $1 \leqq q \leqq \infty)\left(M_{s}, 1 \leqq s \leqq \infty\right)$ if and only if there exists a constant $C$ such that for all $h \in S_{0}(G)$

(2.1) $|\langle h, \sigma\rangle| \leqq C\|h\|_{p^{\prime} q^{\prime}} \quad\left(|\langle h, \sigma\rangle| \leqq C\|h\|_{\infty s^{\prime}}\right)$.

Moreover, if (2.1) holds then

$$
\|\sigma\|_{p q} \leqq C \quad\left(\|\sigma\|_{s} \leqq 2^{a} C\right)
$$

Remark 2.7. From Proposition 2.6 we easily recover what is already known about the Fourier transform of functions in $\left(L^{p}, l^{q}\right)(1 \leqq p, q \leqq 2)$ and measures in $M_{s}(1 \leqq s \leqq 2)$, namely, the Hausdorff-Young theorem for amalgams ( [1, Theorem II], [16, Theorem 8], [21, Theorem 4.2] ). That is,

$$
\left(L^{p}, l^{q}\right)^{\wedge} \subset\left(L^{q^{\prime}}, l^{p^{\prime}}\right), M_{s}^{\wedge} \subset\left(L^{s^{\prime}}, l^{\infty}\right)
$$

and there exists a constant $C$ depending on $G, p$ and $q$ such that

(2.3) $\|\hat{\mu}\|_{s^{\prime} \infty} \leqq C\|\mu\|_{s} \quad(1 \leqq s \leqq 2)$.

Now, since $\left(L^{p}, l^{q}\right) \subset\left(L^{2}, l^{q}\right)$ for $1 \leqq q \leqq 2<p \leqq \infty$, we have that $\left(L^{p}, l^{q}\right)^{\wedge} \subset\left(L^{q^{\prime}}, l^{2}\right)$. So, by $(2.2)$ and $(1.7)$, for $f \in\left(L^{p}, l^{q}\right)$ and $1 \leqq q \leqq 2<p \leqq \infty$,

(2.4) $\|\hat{f}\|_{q^{\prime} 2} \leqq C\|f\|_{p q}$.

By property P.5) we can define $\sigma * f$ for $\sigma \in S_{0}(G)^{*}$ and $f \in L^{1}(G)$ to be an element of $S_{0}(G)^{*}$ given by

$$
\langle h, \sigma * f\rangle=\langle h * f, \sigma\rangle \quad\left(h \in S_{0}(G)\right) .
$$

Moreover, if $g \in L^{1}(\hat{G})$ and $h \in S_{0}(G)$, then $h \stackrel{v}{g}$ belongs to $S_{0}(G)$ because for any $\psi_{\alpha}$ (as in Definition 2.1)

$$
\left\|h \stackrel{v}{g} \psi_{\alpha}\right\|_{A}=\left\|\hat{h} * g * \hat{\psi}_{\alpha}\right\|_{1} \leqq\|g\|_{1}\left\|h \psi_{\alpha}\right\|_{A} .
$$

So we have that

$$
\|h \stackrel{v}{g}\|_{S_{0}} \leqq\|g\|\left\|_{1}\right\| h \|_{S_{0}}
$$

and we can define $\sigma \stackrel{\vee}{g}$ for $\sigma \in S_{0}(G)^{*}, g \in L^{1}(\hat{G})$ to be an element of $S_{0}(G)^{*}$ given by

$$
\langle h, \sigma \stackrel{\vee}{g}\rangle=\langle h \stackrel{\vee}{h}, \sigma\rangle \quad\left(h \in S_{0}(G)\right) .
$$

Proposition 2.8. Let $\sigma \in S_{0}(G)^{*}, f \in L^{1}(G), g \in L^{1}(\hat{G})$. Then

i) $(\sigma * f)^{\wedge}=\hat{\sigma} \hat{f}$

ii) $(\sigma \stackrel{\vee}{g})^{\wedge}=\hat{\sigma} * g$. 
Proof. Let $h \in S_{0}(\hat{G})$. By (2.6) and Definition 2.3 we have that

$$
\begin{aligned}
\langle h, \hat{\sigma} \hat{f}\rangle & =\langle h \hat{f}, \sigma\rangle=\left\langle(h \hat{f})^{v}, \sigma\right\rangle=\left\langle(\stackrel{v}{h} * f)^{\prime}, \sigma\right\rangle=\left\langle h^{\prime} * f, \sigma\right\rangle \\
& =\left\langle\breve{h}^{\prime}, \sigma * f\right\rangle=\left\langle h,(\sigma * f)^{\wedge}\right\rangle .
\end{aligned}
$$

Therefore i) holds. Now, by Remark 2.4 and part i),

$$
(\hat{\sigma} * g)^{\vee}=\sigma \stackrel{\vee}{g}
$$

so

$$
\hat{\sigma} * g=(\sigma \stackrel{\vee}{g})^{\wedge} .
$$

\section{The main theorem.}

Definition 3.1. Let $A$ be a Banach algebra and $B$ be a Banach $A$-module [7]. A continuous linear operator $T: A \rightarrow B$ is a $c$-multiplier from $A$ to $B$ if $T$ commutes with convolution. That is, for all $f, g$ in $A$,

$$
T f * g=T(f * g) \text {. }
$$

The space of $c$-multipliers from $A$ to $B$ will be denoted by $c-M(A, B)$.

THEOREM 3.2. Let $1 \leqq p, q \leqq \infty$. If $B$ is any of the spaces $\left(L^{p}, l^{q}\right)$, $\left(C_{0}, l^{q}\right),\left(L^{p}, c_{0}\right), M_{q}, S$ is any of the algebras $\left(L^{p}, l^{1}\right),\left(C_{0}, l^{1}\right)$, and $T: S \rightarrow B$ is a linear operator, then the following are equivalent:

i) $T \in c-M(S, B)$.

ii) There exists a unique $\sigma \in S_{0}(\hat{G})^{*}$ such that $(T f)^{\wedge}=\hat{\sigma} \hat{f}$ for all $f \in S$.

iii) There exists a unique $\mu \in S_{0}(G)^{*}$ such that $T f=\mu * f$ for all $f \in S$.

Proof. First observe that $S \subset L^{1}$ (see (1.3)) and $B$ is an $L^{1}$-module, hence an $S$-module. By Proposition 2.8 it is clear that ii) is equivalent to iii) with $\sigma=\hat{\mu}$. We will show that i) is equivalent to ii).

Suppose i). If $B$ is any of the spaces $\left(L^{p}, l^{q}\right),\left(L^{p}, c_{0}\right)(1 \leqq p, q<\infty)$ or $\left(C_{0}, l^{s}\right)(1 \leqq s \leqq \infty)$, then $B^{*}$ is either an amalgam space or $M_{s^{\prime}}$. If $B$ is any of the spaces $\left(L^{\infty}, l^{q}\right)(1 \leqq q \leqq \infty),\left(L^{p}, l^{\infty}\right)(1<p \leqq \infty)$, or $M_{s}(1 \leqq s \leqq \infty)$, then $B$ is the dual of an amalgam space $C$. Hence by the Hölder inequality for amalgams (Theorem 1.4)

$$
\begin{array}{ll}
|\langle f, g\rangle| \leqq\|f\|_{B}\|g\|_{B^{*}} & \left(f \in B, g \in \mathrm{B}^{*}\right), \quad \text { and } \\
|\langle f, g\rangle| \leqq\|f\|_{C}\|g\|_{B} \quad(f \in C, g \in B) .
\end{array}
$$

If $B$ is either $\left(L^{\infty}, c_{0}\right)$ or $\left(L^{1}, l^{\infty}\right)$, then $B$ can be considered as a subspace of $M_{\infty}$ (see (1.9)), so again by Theorem 1.4 and (1.7)

$$
\begin{array}{ll}
|\langle f, g\rangle| \leqq\|f\|_{\infty}\|g\|_{1 \infty} & \left(f \in\left(C_{0}, l^{1}\right), g \in\left(L^{1}, l^{\infty}\right)\right) \\
|\langle f, g\rangle| \leqq\|f\|_{\infty}\|g\|_{\infty} & \left(f \in\left(C_{0}, l^{1}\right), g \in\left(L^{\infty} c_{0}\right)\right) .
\end{array}
$$

In either case we conclude by (1.6) and (1.7) that

$$
|\langle f, g\rangle| \leqq\|f\|_{\infty 1}\|g\|_{B} \quad\left(g \in B, f \in\left(C_{0}, l^{1}\right)\right) .
$$


(Remember that $\left(C_{0}, l^{1}\right)$ is included in all amalgam spaces.) For $f, g \in S$, we have that

$$
T f * g=T(f * g)=T g * f .
$$

So

$$
(T f)^{\wedge} \hat{g}=(T g)^{\wedge} \hat{f}
$$

by Proposition 2.8 and this implies by (2.6) that for all $f, g \in S$ and $h \in S_{0}(\hat{G})$,

$$
\left\langle h \hat{g},(T f)^{\wedge}\right\rangle=\left\langle h,(T f)^{\wedge} \hat{g}\right\rangle=\left\langle h,(T g)^{\wedge} \hat{f}\right\rangle=\left\langle h \hat{f},(T g)^{\wedge}\right\rangle .
$$

Let $\left\{\psi_{\alpha}\right\}_{I} \subset A_{c}(\hat{G})$ be as in Definition 2.1 and $W=$ supp $\psi$. To each $\alpha \in I$ we associate a function $\lambda_{\alpha}$ in $\left(C_{0}, l^{1}\right)(G)$ as follows. Take $\lambda_{W}$ in $\left(C_{0}, l^{1}\right)(G)$ such that

$$
\hat{\lambda}_{W} \equiv 1 \text { on } W \text { and } \hat{\lambda}_{W} \in C_{c}(\hat{G})
$$

[21, Theorem 3.1]. Then $\lambda_{\alpha}=[\cdot, \alpha] \lambda_{W}$. It is clear that each $\lambda_{\alpha}$ has the following properties:

1) $\lambda_{\alpha} \in\left(C_{0}, l^{1}\right)(G)$

2) $\hat{\lambda}_{\alpha}(\hat{x})=\hat{\lambda}_{W}(\hat{x}-\alpha)$, hence $\hat{\lambda}_{\alpha} \equiv 1$ on supp $\psi_{\alpha}$.

3) $\hat{\lambda}_{\alpha} \in C_{c}(\hat{G})$, hence $\lambda_{\alpha}=\left(\hat{\lambda}_{\alpha}\right)^{v}$

$$
\text { 4) }\left\|\lambda_{\alpha}\right\|_{\infty 1}=\left\|\lambda_{W}\right\|_{\infty 1} \text {. }
$$

We define $\sigma$ on $S_{0}(\hat{G})$ by

$$
\langle h, \sigma\rangle=\sum\left\langle h \psi_{\alpha},\left(T \lambda_{\alpha}\right)^{\wedge}\right\rangle \quad\left(h \in S_{0}(G)\right) .
$$

First of all, if $h \in S_{0}(\hat{G})$ then $h \psi_{\alpha} \in A_{c}(\hat{G})$ because

$$
h \psi_{\alpha} \in C_{c}(\hat{G}),\left(h \psi_{\alpha}\right)^{v}=\stackrel{\vee}{h} * \check{\psi}_{\alpha} \quad \text { and } \stackrel{v}{h} * \check{\psi}_{\alpha} \in\left(C_{0}, l^{1}\right)(G)
$$

(Theorem 1.6). Also

$$
h \psi_{\alpha}=h \phi_{\alpha} \hat{\lambda}_{\alpha}
$$

by P.2) and this implies that

$$
\begin{aligned}
\left\|\left(h \psi_{\alpha}\right)^{v}\right\|_{\infty 1} & =\left\|\breve{h} * \hat{\psi}_{\alpha} * \lambda_{\alpha}\right\|_{\infty 1} \leqq\left\|\lambda_{\alpha}\right\|_{\infty 1}\left\|\breve{h} * \check{\psi}_{\alpha}\right\|_{1} \\
& =\left\|\lambda_{W}\right\|_{\infty 1}\left\|h \psi_{\alpha}\right\|_{A} .
\end{aligned}
$$

Therefore by (3.1) and (1.7)

$$
\begin{aligned}
\left|\left\langle h \psi_{\alpha},\left(T \lambda_{\alpha}\right)^{\wedge}\right\rangle\right| & =\left|\left\langle\left(h \psi_{\alpha}\right)^{\wedge},\left(T \lambda_{\alpha}\right)\right\rangle\right| \leqq\left\|T \lambda_{\alpha}\right\|_{B}\left\|\left(h \psi_{\alpha}\right)^{v}\right\|_{\infty 1} \\
& \leqq\|T\|\left\|\lambda_{W}\right\|_{S}\left\|\lambda_{W}\right\|_{\infty 1}\left\|h \psi_{\alpha}\right\|_{A} \\
& \leqq\|T\|\left\|\lambda_{W}\right\|_{\infty}^{2}\left\|h \psi_{\alpha}\right\|_{A} .
\end{aligned}
$$

Hence $\sigma$ is well-defined and for all $h \in S_{0}(\hat{G})$ 


$$
|\langle h, \sigma\rangle| \leqq\|T\|\left\|\lambda_{W}\right\|_{\infty 1}^{2}\|h\|_{S_{0}} .
$$

If $\xi_{W}$ is another function in $\left(C_{0}, l^{l}\right)(G)$ with the same properties as $\lambda_{W}$ and $\xi_{\alpha}=[\cdot, \alpha] \xi_{W}$, then by (3.2) we have that for all $h \in S_{0}(\hat{G})$

$$
\begin{aligned}
\left\langle h \psi_{\alpha},\left(T \lambda_{\alpha}\right)^{\wedge}\right\rangle & =\left\langle h \psi_{\alpha} \hat{\xi}_{\alpha},\left(T \lambda_{\alpha}\right)^{\wedge}\right\rangle \\
& =\left\langle h \psi_{\alpha} \hat{\lambda}_{\alpha},\left(T \xi_{\alpha}\right)^{\wedge}\right\rangle=\left\langle h \psi_{\alpha},\left(T \xi_{\alpha}\right)^{\wedge}\right\rangle .
\end{aligned}
$$

Hence $\sigma$ is independent of the choice of the function $\lambda_{W}$.

Now, if $h \in A_{E}(\hat{G})$, then

$$
\left\{h \psi_{\alpha}\right\}_{I} \subset A_{c}(\hat{G}), h \psi_{\alpha}=0
$$

for all but finitely many $\alpha$ 's and $h=\sum h \psi_{\alpha}$ pointwise by P.3). Then, for $f \in S$,

$$
\left\langle h,(T f)^{\wedge}\right\rangle=\Sigma\left\langle h \psi_{\alpha},(T f)^{\wedge}\right\rangle
$$

this together with (3.2) and (2.6) implies that for $f \in S$ and $h \in A_{c}(\hat{G})$

$$
\begin{aligned}
\langle h, \sigma \hat{f}\rangle & =\langle h \hat{f}, \sigma\rangle=\sum\left\langle h \psi_{\alpha} \hat{f},\left(T \lambda_{\alpha}\right)^{\wedge}\right\rangle=\sum\left\langle h \psi_{\alpha} \hat{\lambda}_{\alpha},(T f)^{\wedge}\right\rangle \\
& =\sum\left\langle h \psi_{\alpha},(T f)^{\wedge}\right\rangle=\left\langle h,(T f)^{\wedge}\right\rangle .
\end{aligned}
$$

Since $A_{c}(\hat{G})$ is dense in $S_{0}(\hat{G})$ we conclude that $(T f)^{\wedge}=\sigma \hat{f}$ for all $f \in S$.

On the other hand, if $h \in A_{E}(\hat{G})$ and $\lambda_{E}$ is a function in $\left(C_{0}, l^{1}\right)(G)$ such that $\hat{\lambda}_{E} \equiv 1$ on $E$ then we have that

$$
\langle h, \sigma\rangle=\left\langle h \hat{\lambda}_{E}, \sigma\right\rangle=\left\langle h, \sigma \hat{\lambda}_{E}\right\rangle=\left\langle h,\left(T \lambda_{E}\right)^{\wedge}\right\rangle .
$$

Finally if $\sigma^{\prime}$ is another element of $S_{o}(\hat{G})^{*}$ such that

$$
(T f)^{\wedge}=\sigma^{\prime} \hat{f} \quad \text { for all } f \in S,
$$

then by (3.3) for all $h \in A_{E}(\hat{G})$

$$
\langle h, \sigma\rangle=\left\langle h,\left(T \lambda_{E}\right)^{\wedge}\right\rangle=\left\langle h, \sigma^{\prime} \hat{\lambda}_{E}\right\rangle=\left\langle h \hat{\lambda}_{E}, \sigma^{\prime}\right\rangle=\left\langle h, \sigma^{\prime}\right\rangle .
$$

Again by P.6), $\sigma=\sigma^{\prime}$ and therefore i) implies ii).

Conversely if ii) holds, then by Proposition 2.8, for $f, g \in S$, we have that

$$
(T(f * g))^{\wedge}=\sigma(f * g)^{\wedge}=(\sigma \hat{f}) \hat{g}=(T f)^{\wedge} \hat{g}=(T f * g)^{\wedge} .
$$

Therefore $T$ commutes with convolution by Remark 2.4. Finally an application of the Closed Graph theorem implies that $T$ is continuous and the proof is complete.

COROllary 3.3. Let $S$ be as in Theorem 3.2 and $B$ be any of the spaces $\left(L^{p}, l^{q}\right),\left(C_{0}, l^{q}\right), M_{q}(1 \leqq p \leqq \infty, 1 \leqq q \leqq 2)$. If $T: S \rightarrow B$ is a linear operator, then the following are equivalent:

i) $T \in c-M(S, B)$.

ii) There exists a unique $\varphi \in\left(L^{q^{\prime}}, l^{\infty}\right)(\hat{G})$ such that

$$
(T f)^{\wedge}=\varphi \hat{f} \quad \text { for all } f \in S
$$



fine

Proof. Let $y \in \hat{G}$ and $\psi_{y}$ be a function in $S$ such that $\hat{\psi}_{y}(y)=1$. De-

$$
\varphi(y)=\left(T \psi_{y}\right)^{\wedge}(y) \quad(y \in \hat{G}) .
$$

If $T \in c-M(S, B)$ then $\varphi$ is independent of the choice of $\psi_{y}$. Indeed if $\xi_{y}$ is another function such that $\hat{\xi}_{y}(y)=1$ then

$$
\begin{aligned}
\left(T \psi_{y}\right)^{\wedge}(y) & =\left(T \psi_{y}\right)^{\wedge}(y) \hat{\xi}_{y}(y)=\left(T \psi_{y} * \xi_{y}\right)^{\wedge}(y) \\
& =\left(T \xi_{y} * \psi_{y}\right)^{\wedge}(y)=\left(T \xi_{y}\right)^{\wedge}(y) \hat{\psi}_{y}(y)=\left(T \xi_{y}\right)^{\wedge}(y) .
\end{aligned}
$$

Also for $f \in S$ and $y \in \hat{G}$

$$
\varphi \hat{f}(y)=\left(T \psi_{y}\right)^{\wedge}(y) \hat{f}(y)=(T f)^{\wedge}(y) \hat{\psi}_{y}(y)=(T f)^{\wedge}(y) .
$$

Let $\sigma$ be the element in $S_{0}(\hat{G})^{*}$ associated to $T$ by Theorem 3.2. Then by (3.3) for $h \in A_{E}(\hat{G})$

$$
\begin{aligned}
\langle h, \sigma\rangle & =\left\langle h,\left(T \lambda_{E}\right)^{\wedge}\right\rangle=\int_{\hat{G}} h(y)\left(T \lambda_{E}\right)^{\wedge}(y) d y \\
& =\int_{E} h(y) \varphi(y) \hat{\lambda}_{E}(y) d y=\int h(y) \varphi(y) d y .
\end{aligned}
$$

Therefore

$$
\langle h, \boldsymbol{\sigma}\rangle=\int_{\hat{G}} h(y) \varphi(y) d y \quad \text { for all } h \in A_{c}(\hat{G}) .
$$

Now take $f \in C_{c}(\hat{G})$ such that $f \equiv 1$ on $\hat{L}$ and $\tilde{f} \in S$ [21, Theorem 3.1]. Then for $\beta \in I$, the function $f_{\beta}$ belongs to $C_{c}(\hat{G}), f_{\beta} \equiv 1$ on $L_{\beta}$, $\stackrel{v}{f}_{\beta}=[\cdot, \beta] \breve{f}$ belongs to $S$, and

$$
\left\|\check{f}_{\beta}\right\|_{S}=\|\check{v}\|_{S}
$$

(see Remark 1.3). By the definition of the norm $\|\cdot\|_{q^{\prime} r}, 1 \leqq r \leqq \infty$, it is clear that

$$
\left\|\boldsymbol{\varphi} \chi_{L_{\beta}}\right\|_{q^{\prime}} \leqq\left\|\boldsymbol{~} f_{\beta}\right\|_{q^{\prime} r} \quad(1 \leqq r \leqq \infty) .
$$

So by (2.2), (2.3) and (2.4) of Remark 2.7 we have that

$$
\left\|\varphi \chi_{L_{\beta}}\right\|_{q^{\prime}} \leqq C\|T\|\|\stackrel{v}{f}\|_{S} .
$$

To see this note that

$$
\left\|_{\varphi} f_{\beta}\right\|_{q^{\prime} r}=\left\|\varphi \hat{f}_{\beta}\right\|_{q^{\prime} r}=\left\|\left(T \tilde{f}_{\beta}\right)^{\wedge}\right\|_{q^{\prime} r} \quad(1 \leqq r \leqq \infty) .
$$

Since this holds for all $\beta \in I$, we conclude that

$$
\varphi \in\left(L^{q^{\prime}}, l^{\infty}\right)(\hat{G}) \text {. }
$$

Hence i) implies ii).

Conversely if ii) holds, then we define $\sigma$ on $A_{c}(\hat{G})$ by 


$$
\langle h, \sigma\rangle=\int_{\hat{G}} h(y) \varphi(y) d y \quad\left(h \in A_{c}(\hat{G})\right) .
$$

Then as above

$$
\langle h, \sigma\rangle=\left\langle h,\left(T \lambda_{E}\right)^{\wedge}\right\rangle \text { for } h \in A_{E}(\hat{G}) .
$$

Since $A_{c}(\hat{G})$ is dense in $S_{0}(\hat{G})$ this implies that $\sigma$ is the element in $S_{0}(\hat{G})^{*}$ given by Theorem 3.2 (see the proof of Theorem 3.2) and therefore ii) implies i).

Let $B$ be a linear space of functions on $G$. If $f_{s} \in B$ for all $f \in B$ and for all $s \in G$ then $B$ is said to be translation invariant. If $B$ is translation invariant the linear operator $f \rightarrow f_{s}(s \in G)$ is called a translation operator. It is easy to see that all amalgams and all $M_{q}$ spaces are translation invariant.

Definition 3.4. Let $A, B$ be two translation invariant spaces. A multiplier from $A$ to $B$ is a bounded linear operator $T: A \rightarrow B$ such that $T$ commutes with translations. That is, $T f_{s}=(T f)_{s}$ for all $s \in G$ and $f \in A$. The space of multipliers from $A$ to $B$ will be denoted by $M(A, B)$.

Let $\left(L^{\infty}, l^{1}\right)^{w}$ be the amalgam $\left(L^{\infty}, l^{1}\right)$ endowed with the weak*topology induced by $\left(L^{1}, c_{0}\right)$ (see Theorem 1.4). Hence

$$
\left(L^{\infty}, l^{1}\right)^{w *}=\left(L^{1}, c_{0}\right)
$$

via the formula

$$
\langle f, g\rangle=\int f(-t) g(t) d t \quad\left(f \in\left(L^{\infty}, l^{1}\right), g \in\left(L^{1}, c_{0}\right)\right)
$$

$[17,5.17 .6]$. The space $M_{1}^{w}$ is defined similarly and therefore $M_{1}^{w *}=\mathrm{C}_{0}$.

A relation between multipliers and $c$-multipliers is given in the next result.

Proposition 3.5. Let $S$ be any algebra $\left(L^{p}, l^{1}\right)(1 \leqq p<\infty),\left(C_{0}, l^{1}\right)$, $\left(L^{\infty}, l^{1}\right)^{w}$ or $M_{1}^{w}$, and $B$ be as in Theorem 3.2. Then

$$
M(S, B) \subset c-M(S, B) .
$$

Proof. An easy calculation shows that for $f, g \in S$ and $\psi \in S^{*}$,

$$
\langle f * g, \psi\rangle=\int g(s)\left\langle f_{s}, \psi\right\rangle d s\left(=\int\left\langle f_{s}, \psi\right\rangle d g(s) \text { if } S=M_{1}^{w}\right) .
$$

If $B$ is any of the spaces $\left(L^{p}, l^{q}\right),\left(L^{p}, c_{0}\right)(1 \leqq p, q<\infty),\left(C_{0}, l^{s}\right)$ $(1 \leqq s \leqq \infty)$, then $B^{*}$ is an amalgam space or $M_{s^{\prime}}$. So for $h \in S, k \in B$, and $F \in B^{*}$

$$
\langle k * h, F\rangle=\int h(s)\left\langle k_{s}, F\right\rangle d s\left(=\int\left\langle k_{s}, F\right\rangle d h(s) \quad \text { if } S=M_{1}^{n}\right) .
$$


If $B$ is any of the spaces $\left(L^{p}, l^{\infty}\right)(1<p \leqq \infty),\left(L^{\infty}, l^{s}\right)$ or $M_{s}(1 \leqq s \leqq \infty)$, then $B=C^{*}$ for some amalgam space $C$. Then (3.5) holds with $h \in S$, $k \in B$ and $F \in C$. If $B=\left(L^{1}, l^{\infty}\right)$ then (3.5) holds with $h \in S, k \in B$ and $F \in C, C=\left(C_{0}, l^{1}\right)$ (think of $\left(L^{1}, l^{\infty}\right)$ as a subspace of $\left.M_{\infty}\right)$. Hence for $F \in B^{*}(F \in C)$ the map

$$
\left\langle f, \Lambda_{F}\right\rangle=\langle T f, F\rangle \quad(f \in S)
$$

belongs to $S^{*}$. So, (3.4), (3.5) and (3.6) imply that for $f, g \in S, F \in B^{*}$ $(F \in C)$

$$
\begin{aligned}
& \langle T f * g, F\rangle \\
& =\int g(s)\left\langle(T f)_{s}, F\right\rangle d s \quad\left(=\int\left\langle(T f)_{s}, F\right\rangle d g(s) \text { if } S=M_{1}{ }^{w}\right) \\
& =\int g(s)\left\langle T f_{s}, F\right\rangle d s \quad\left(=\int\left\langle T f_{s}, F\right\rangle d g(s)\right) \\
& =\int g(s)\left\langle f_{s}, \Lambda_{F}\right\rangle d s \quad\left(=\int\left\langle f_{s}, \Lambda_{F}\right\rangle d g(s)\right) \\
& =\left\langle f * g, \Lambda_{F}\right\rangle=\langle T(f * g), F\rangle .
\end{aligned}
$$

Therefore $T$ commutes with convolution.

TheOREM 3.6. Let $A$ be any of the spaces $\left(L^{p}, l^{q}\right),\left(C_{0}, l^{q}\right)(1 \leqq p<\infty$, $1 \leqq q \leqq 2)$ and let $B$ be any of the spaces $\left(L^{r}, l^{s}\right),\left(C_{0}, l^{s}\right)(1 \leqq r<\infty$, $1 \leqq s \leqq 2$ ). If $T: A \rightarrow B$ is a linear operator, then i) implies ii).

i) $T \in M(A, B)$.

ii) There exists a unique $\varphi \in\left(L^{s^{\prime}}, l^{\infty}\right)(\hat{G})$ such that

$$
(T f)^{\wedge}=\varphi \hat{f} \quad \text { for all } f \in A .
$$

Proof. We will prove the theorem for

$$
\mathrm{A}=\left(L^{p}, l^{q}\right) \text { and } B=\left(L^{r}, l^{s}\right) \quad(1 \leqq r, s \leqq 2) .
$$

The remaining cases are similar.

Suppose $T \in M(A, B)$. Then $T \mid\left(L^{p}, l^{1}\right)$ belongs to $c-M\left(\left(L^{p}, l^{1}\right), B\right)$ by Proposition 3.5. So by Corollary 3.3 there exists a unique $\varphi$ in $\left(L^{s^{\prime}}, l^{\infty}\right)(\hat{G})$ such that

$$
(T f)^{\wedge}=\varphi \hat{f} \quad \text { for all } f \in\left(L^{p}, l^{1}\right) .
$$

Now by (2.2) for $f \in\left(L^{p}, l^{1}\right)$ we have that

$$
\|\varphi \hat{f}\|_{s^{\prime} r^{\prime}}=\left\|(T f)^{\wedge}\right\|_{s^{\prime} r^{\prime}} \leqq C\|T f\|_{r s} \leqq C\|T\|\|f\|_{p q} .
$$

Therefore the map $f \rightarrow \varphi \hat{f}$ is continuous on $\left(\left(L^{p}, l^{q}\right),\|\cdot\|_{p q} \|\right)$. Since $\left(L^{p}, l^{1}\right)$ is dense in $\left(L^{p}, l^{q}\right)([2$, Section $7 \mathrm{e})]$ and $\left.(1.1)\right)$ this map has a unique continuous extension on $\left(L^{p}, l^{q}\right)$ and this implies that 


$$
(T f)^{\wedge}=\varphi \hat{f} \quad \text { for all } f \in\left(L^{p}, l^{q}\right)
$$

4. Equivalent norms. We need to introduce now, several equivalent norms in order to characterize the space of $c$-multipliers from $L^{1}$ to amalgams and $M_{q}$ spaces.

Since $\left(L^{p}, l^{q}\right)(1 \leqq p, q \leqq \infty)$ is an $L^{1}$-module there exists an equivalent norm $\|\cdot\|_{p q}^{\prime}$ such that

$$
\|f * \mu\|_{p q}^{\prime} \leqq\|\mathbf{f}\|_{1}\|\mu\|_{p q}^{\prime} \quad\left(f \in L^{1}, \mu \in\left(L^{p}, l^{q}\right)\right) .
$$

and

$$
\|\cdot\|_{p q} \leqq\|\cdot\|_{p q}^{\prime} \leqq 2^{a}\|\cdot\|_{p q}
$$

[7, (4.14) ]. The amalgam $\left(L^{p}, l^{q}\right)$ endowed with the norm $\|\cdot\|_{p q}^{\prime}$ will be denoted by $\left(L^{p}, l^{q}\right)^{\prime}$.

Let $\left\{e_{\alpha}\right\}$ be an a.i. in $L^{1}$ such that

$$
\left\|e_{\alpha}\right\|_{1}=1 \text { for all } \alpha \text {. }
$$

For $\mu \in\left(L^{p}, l^{q}\right)(1 \leqq p, q \leqq \infty)$ we define

(4.2) $\|\mu \mu\|\left\|_{p q}=\sup _{\alpha}\right\| \mu * e_{\alpha} \|_{p q}^{\prime}$.

It is clear from (4.1) that $\||\cdot|\|_{p q}$ is well defined and that

$$
\||\cdot|\|_{p q} \leqq\|\cdot\|_{p q}^{\prime} \text {. }
$$

Now by Theorem 1.4 if $\mu \in\left(L^{p}, l^{q}\right)(1<p \leqq \infty, 1 \leqq q \leqq \infty)$ then

$$
\|\mu\|_{p q}=\sup \left\{|\langle\phi, \mu\rangle| ; \phi \in\left(L^{p^{\prime}}, l^{q^{\prime}}\right),\|\phi\|_{p^{\prime} q^{\prime}} \leqq 1\right\}
$$

if $q \neq 1$, and

$$
\|\mu\|_{p 1}=\sup \left\{|\langle\phi, \mu\rangle| ; \phi \in\left(L^{p^{\prime}}, c_{0}\right),\|\phi\|_{p^{\prime} \infty} \leqq 1\right\} .
$$

Let $\phi \in\left(L^{p^{\prime}}, l^{q^{\prime}}\right)\left(\left(L^{p^{\prime}}, c_{0}\right)\right.$ if $\left.q=1\right)$ such that

$$
\|\phi\|_{p^{\prime} q^{\prime}} \leqq 1 \text {. }
$$

By Proposition 1.8 and Theorem 1.4 we have that

$$
\begin{aligned}
|\langle\phi, \mu\rangle| & =\lim \left|\left\langle\phi * e_{\alpha}, \mu\right\rangle\right|=\lim \left|\left\langle\phi, \mu * e_{\alpha}\right\rangle\right| \\
& \leqq\|\phi\|_{p^{\prime} q^{\prime}} \lim \left\|\mu * e_{\alpha}\right\|_{p q}^{\prime} \\
& \leqq\|\mu\| \|_{p q} .
\end{aligned}
$$

Therefore

$$
\|\mu\|_{p q} \leqq\|\mu \mid\|_{p q}
$$

and this means that $\||\cdot|\|_{p q}$ is an equivalent norm in $\left(L^{p}, l^{q}\right)(1<p \leqq \infty$, $1 \leqq q \leqq \infty)$. We will denote by $\left(L^{p}, l^{q}\right)^{\sim}$ the amalgam $\left(L^{p}, l^{q}\right)$ endowed with the norm $\||\cdot|\|_{p q}$. 
Finally we introduce in the next theorem an equivalent norm originally defined in [2, Proposition VIII] (see also [18] ). For a complete proof see [22, Theorem 1.21].

TheOREM 4.1. i) Let $1 \leqq p, q \leqq \infty$. A function $f$ belongs to $\left(L^{p}, l^{q}\right)$ $\left(\left(L^{p}, c_{0}\right)\right)$ if and only if the function $f^{\#}$ on $G$ defined by

$$
f^{\#}(t)=\|f\|_{L^{p}(t+L)}
$$

belongs to $L^{q}\left(C_{0}\right)$. If

$$
\|f\|_{p q}^{\#}=\left\|f^{\#}\right\|_{q}
$$

then

$$
2^{-a}\|f\|_{p q} \leqq\|f\|_{p q}^{\#} \leqq 2^{a}\|f\|_{p q} .
$$

ii) Let $1 \leqq q \leqq \infty$. A measure $\mu$ belongs to $M_{q}$ if and only if the function $\mu^{\#}$ defined by

$$
\mu^{\#}(t)=|\mu|(t+L)
$$

belongs to $L^{q}$. If $\|\mu\|_{q}^{\#}=\left\|\mu^{\#}\right\|_{q}$, then

$$
2^{-a}\|\mu\|_{a} \leqq\|\mu\|_{q}^{\#} \leqq 2^{a}\|\mu\|_{q} \text {. }
$$

The amalgam $\left(L^{p}, l^{q}\right)$ endowed with the norm $\|\cdot\|_{p q}^{\#}$ will be denoted by $\left(L^{p}, l^{q}\right)^{\#}$. Similarly for $M_{q}^{\#}$.

The next result is a direct consequence of the definition of $\|\cdot\|_{q}^{\#}$.

Proposition 4.2. [22, Corollary 4.6]. If $f \in L^{1}(G)$ and $\mu \in M_{q}(G)$ $(1 \leqq q \leqq \infty)$, then

$$
f * \mu \in\left(L^{1}, l^{q}\right) \text { and }\|f * \mu\|_{1 q}^{\#} \leqq\|f\|_{1}\|\mu\|_{q}^{\#} .
$$

5. c-multipliers from $L^{1}$ to amalgams and $M_{q}$ spaces. Because of Theorem 3.2 in each one of the following theorems it will be enough to show that the " $\mu$ " given by Theorem 5.1 belongs to the corresponding space, and establish the isometric isomorphism.

In this section $\left\{e_{n}\right\}$ will be an a.i. in $S_{0}(G)$ (hence in $L^{1}(G)$ ) such that $\left\|e_{n}\right\|_{1}=1$ for all $n$.

THEOREM 5.1. Let $1<p, q<\infty, 1 \leqq s \leqq \infty$ and let $B$ be any of the spaces $\left(L^{p}, l^{q}\right),\left(L^{p}, l^{\infty}\right),\left(L^{\infty}, l^{s}\right)$. If $T: L^{1} \rightarrow B$ is a linear operator, then the following are equivalent:

i) $T \in c-M\left(L^{1}, B\right)$.

ii) There exists a unique $\mu \in B$ such that $T f=\mu *$ f for all $f \in L^{1}$.

iii) There exists a unique $\sigma \in S_{0}(\hat{G})^{*}(\sigma=\hat{\mu})$ such that

$$
(T f)^{\wedge}=\sigma \hat{f} \text { for all } f \in L^{1} \text {. }
$$


The correspondence between $T$ and $\mu$ establishes an isometric isomorphism from $c-M\left(L^{1},\left(L^{p}, l^{q}\right)^{\prime}\right)$ onto $\left(L^{p}, l^{q}\right)^{\prime} ; c-M\left(L^{1},\left(L^{p}, l^{\infty}\right)^{\prime}\right)$ onto $\left(L^{p}, l^{\infty}\right)^{\sim} ; c-M\left(L^{1},\left(L^{\infty}, l^{s}\right)^{\prime}\right)$ onto $\left(L^{\infty}, l^{s}\right)^{\sim}$.

Proof. We will prove the first part of the theorem for $B=\left(L^{p}, l^{q}\right)$, the remaining cases are proved similarly (remember that $\left(L^{1}, c_{0}\right)^{*}=$ $\left.\left(L^{\infty}, l^{1}\right)\right)$. Let $\mu$ be the element in $S_{0}(G)^{*}$ given by Theorem 3.2 , then for $h$ in $S_{0}(G)$

$$
\begin{aligned}
|\langle h, \mu\rangle| & =\lim \left|\left\langle h * e_{n}, \mu\right\rangle\right| \\
& =\lim \left|\left\langle h, \mu * e_{n}\right\rangle\right|=\lim \left|\left\langle h, T e_{n}\right\rangle\right| \\
& \leqq\|h\|_{p^{\prime} q^{\prime}} \lim \left\|T e_{n}\right\|_{p q} \leqq\|T\|\|h\|_{p^{\prime} q^{\prime}}
\end{aligned}
$$

Hence $\mu \in\left(L^{p}, l^{q}\right)$ by Proposition 2.6, and if

$$
T \in c-M\left(L^{1},\left(L^{p}, l^{q}\right)^{\prime}\right),
$$

then

$$
\|T\| \leqq\|\mu\|_{p q}^{\prime}
$$

by (4.1).

On the other hand, by Proposition 1.8,

$$
\lim \left\|\mu * e_{n}-\mu\right\|_{p q}=0 .
$$

So given $\epsilon>0$ there exists $N$ such that

$$
\left\|\mu * e_{N}-\mu\right\|_{p q}^{\prime}<\epsilon .
$$

So

$$
\left\|T e_{N}\right\|_{p q}^{\prime}=\left\|\mu * e_{N}\right\|_{p q}^{\prime}>\|\mu\|_{p q}^{\prime}-\epsilon
$$

and this implies that

$$
\|\mu\|_{p q}^{\prime} \leqq\|T\| \text {. }
$$

If $T \in c-M\left(L^{l},\left(L^{p}, l^{\infty}\right)^{\prime}\right)$ then

$$
\|\mu\|\left\|_{p \infty} \leqq \sup \right\| T e_{n}\left\|_{p \infty}^{\prime} \leqq\right\| T\|\sup \| e_{n}\left\|_{1}=\right\| T \| .
$$

Now, for $f \in L^{1}$

$$
\begin{aligned}
\|T f\|_{p \infty}^{\prime} & =\lim \left\|T f * e_{n}\right\|_{p \infty}^{\prime}=\lim \left\|f * \mu * e_{n}\right\|_{p \infty}^{\prime} \\
& \leqq\|f\|_{1} \lim \left\|\mu * e_{n}\right\|_{p \infty}^{\prime} \\
& =\|f\|_{1}\|\mu\| \|_{p \infty} .
\end{aligned}
$$

Hence

$$
\|T\| \leqq\|\mu\| \|_{p \infty}
$$

The proof for $B=\left(L^{\infty}, l^{s}\right)$ is similar to the previous case. 
THEOREM 5.2. Let $1 \leqq q \leqq \infty$ and let $B$ either $\left(L^{1}, l^{q}\right)$ or $M_{q}$. If $T: L^{1} \rightarrow B$ is a linear operator then the following are equivalent:

i) $T \in c-M\left(L^{1}, B\right)$.

ii) There exists a unique $\mu \in M_{q_{\hat{N}}}$ such that $T f=\mu *$ ffor all $f \in L^{1}$.

iii) There exists a unique $\sigma \in S(\hat{G})^{*}(\sigma=\hat{\mu})$ such that

$$
(T f)^{\wedge}=\sigma \hat{f} \quad \text { for all } f \in L^{1} .
$$

The correspondence between $T$ and $\mu$ establishes an isometric isomorphism from $c-M\left(L^{1}, M_{q}^{\#}\right)$ onto $M_{q}^{\#}$ and

$$
c-M\left(L^{1},\left(L^{1}, l^{q}\right)^{\#}\right)=c-M\left(L^{1}, M_{q}^{\#}\right) .
$$

Proof. The first part is the same as the first part of Theorem 5.1.

For $B=M_{\infty}^{\#}$ the second part is [9, Theorem 1.3]. It should be mentioned here that the definition of a multiplier used throughout [9] corresponds to what we call a $c$-multiplier and not the one given in $[9$, p. 342].

If $\mu \in M_{q}(1 \leqq q \leqq \infty)$ and $T f=\mu * f$ for all $f \in L^{1}$, then

$$
\|T\| \leqq\|\mu\|_{q}^{\#}
$$

by Proposition 4.2. Since $\mu^{\#} \in L^{q}$ and $q$ is finite, given $\epsilon>0$ there exists a neighborhood $U$ of 0 such that

$$
\left\|h * \mu^{\#}-\mu^{\#}\right\|_{q}<\epsilon
$$

for all $h \in L^{1},\|h\|_{1}=1, h \geqq 0$ and

$$
\int_{G \sim U} h=0
$$

[15, Theorem 20.15]. Clearly the function

$$
f=1 / m(U) \cdot \chi_{U}
$$

satisfies all the above conditions and an easy calculation shows that

$$
(f * \mu)^{\#}=f * \mu^{\#} \text {. }
$$

So we have that

$$
\|T f\|_{q}^{\#}=\|f * \mu\|_{q}^{\#}=\left\|(f * \mu)^{\#}\right\|_{q}=\left\|f * \mu^{\#}\right\|_{q}>\|\mu\|_{q}^{\#}-\epsilon .
$$

Therefore

$$
\|T\| \geqq\|\mu\|_{q}^{\#} .
$$

Finally,

$$
L^{1} * M_{q} \subset\left(L^{1}, l^{q}\right)(1 \leqq q \leqq \infty)
$$

by Theorem 1.6, hence

$$
c-M\left(L^{1}, M_{q}\right) \subset c-M\left(L^{1},\left(L^{1}, l^{q}\right)\right),
$$


this together with (1.9) implies the rest of the theorem.

Remark 5.3. Theorem 5.2 for $B=\left(L^{1}, l^{q}\right)$ is already known [18, Corollary 6.3] and it can also be deduced from [9, Theorem 1.5].

The next theorem was proved for $q=1$ by Burnham and Goldberg [4, Theorem 4.6] using a different method. Its proof is the same as that of Theorem 5.1.

THEOREM 5.4. Let $1 \leqq q<\infty$. If

$$
T: L^{1} \rightarrow\left(C_{0}, l^{q}\right)
$$

is a linear operator, then the following are equivalent:

i) $T \in c-M\left(L^{1},\left(C_{0}, l^{q}\right)\right)$.

ii) There exists a unique $\mu \in\left(L^{\infty}, l^{q}\right)$ such that

$$
T f=\mu * f \text { for all } f \in L^{1} .
$$

iii) There exists a unique $\sigma \in S(\hat{G})^{*}(\sigma=\hat{\mu})$ such that

$$
(T f)^{\wedge}=\sigma \hat{f} \text { for all } f \in L^{1} .
$$

The correspondence between $T$ and $\mu$ establishes an isometric isomorphism from $c-M\left(L^{1},\left(C_{0}, l^{q}\right)^{\prime}\right)$ onto $\left(C_{0}, l^{q}\right)^{\sim}$.

To characterize the space $c-M\left(L^{1},\left(L^{p}, c_{0}\right)\right)(1 \leqq p<\infty)$ we use Feichtinger's results in [9]. First we see that $\left(L^{p}, c_{0}\right)(1 \leqq p<\infty)$ is a homogeneous Banach space (as in [9, p. 342]).

1) Since $\left(L^{p}, c_{0}\right) \subset\left(L^{1}, c_{0}\right)$ we have that

$$
\left(L^{p}, c_{0}\right) \subset L_{\text {loc }}^{1}
$$

2) $\left(L^{p}, c_{0}\right)$ is translation invariant and by Theorem 1.5 the map $s \mapsto f_{s}$ is continuous on $G$ for all $f \in\left(L^{p}, c_{0}\right)$.

3) For $s \in G$ and $f \in\left(L^{p}, c_{0}\right)$ we have that

$$
\left\|f_{s}\right\|_{p}^{\#}=\left\|\left(f_{s}\right)^{\#}\right\|_{\infty}=\left\|\left(f^{\#}\right)_{-s}\right\|_{\infty}=\left\|f^{\#}\right\|_{\infty}=\|f\|_{p \infty}^{\#}
$$

4) Convergence in $\left(L^{p}, c_{0}\right)$ implies convergence in measure. Indeed, let $f,\left\{f_{n}\right\}$, be in $\left(L^{p}, c_{0}\right)$ such that

$$
\lim \left\|f_{n}-f\right\|_{p \infty}=0 \text {. }
$$

Since $\left(f_{n}-f\right)^{\#}$ belongs to $C_{0}$, given $\epsilon>0$ there exists a compact set $E \subset G$ such that

$$
\left\|f_{n}-f\right\|_{L^{p}(x+L)}<\epsilon \text { for all } x \notin E .
$$

Let

$$
E_{n}(\epsilon)=\left\{x|| f_{n}(x)=f(x) \mid \geqq \epsilon\right\}
$$

and suppose that

$$
(G \sim E) \cap\left(E_{n}(\epsilon)-L\right) \neq \emptyset .
$$


So for $x$ in this intersection

$$
x+L \subset E_{n}(\epsilon),
$$

hence

$$
\begin{aligned}
\epsilon>\left(f_{n}-f\right)^{\#}(x) & =\left(\int_{x+L}\left|f_{n}(t)-f(t)\right|^{p}\right)^{1 / p} \\
& \geqq \epsilon m(x+L)^{1 / p}=\epsilon .
\end{aligned}
$$

This contradiction implies that $E_{n}(\epsilon) \subset E+L$ and therefore the cardinality of the set

$$
F=\left\{\alpha \mid E_{n}(\epsilon) \cap L_{\alpha} \neq \emptyset\right\}
$$

is finite [22, p. 35]. So

$$
\left\|f_{n}-f\right\|_{p \infty} \geqq\left\|f_{n}-f\right\|_{L^{p}\left(L_{\alpha} \cap E_{n}(\epsilon)\right)} \geqq \epsilon m\left(L_{\alpha} \cap E_{n}(\epsilon)\right)^{1 / p} .
$$

Then,

$$
0=\lim \left\|f_{n}-f\right\|_{p \infty} \geqq \epsilon \lim m\left(L_{\alpha} \cap E_{n}(\epsilon)\right)^{1 / p}
$$

for all $\alpha \in J$. Since

$$
m\left(E_{n}(\epsilon)\right)=\sum_{F} m\left(E_{n}(\epsilon) \cap L_{\alpha}\right)
$$

we conclude that

$$
m\left(E_{n}(\epsilon)\right)=0 .
$$

Moreover $\left(L^{p}, c_{0}\right)(1 \leqq p<\infty)$ is an essential $C_{0}(G)$-module [2, Section 7, e)] and [9, Lemma 2.6]. This implies the following theorem where, in the notation of [9],

$$
\left(L^{p}, c_{0}\right)_{4}^{\widetilde{T}}=\left\{\mu \in V(G) \mid\left\{e_{n} * \mu\right\} \text { is bounded in }\left(L^{p}, c_{0}\right)\right\}
$$

endowed with the norm

$$
\|\mu\|\|=\sup \| e_{n} * \mu \|_{p \infty}^{\#}
$$

$\left\{e_{n}\right\}$ being an a.i. in $L^{1}$.

TheOrem 5.5. Let $1 \leqq p<\infty$. If

$$
T: L^{1} \rightarrow\left(L^{p}, c_{0}\right)
$$

is a linear operator, then the following are equivalent:

i) $T \in c-M\left(L^{1},\left(L^{p}, c_{0}\right)\right)$.

ii) There exists a unique $\mu \in\left(L^{p}, c_{0}\right)$ such that

$$
T f=\mu * f \text { for all } f \in L^{1} .
$$


iii) There exists a unique $\left.\sigma \in S_{0} \hat{G}\right)^{*}(\sigma=\hat{\mu})$ such that

$$
(T f)^{\wedge}=\sigma \hat{f} \text { for all } f \in L^{1} \text {. }
$$

The correspondence between $T$ and $\mu$ establishes an isometric isomorphism from $c-M\left(L^{1},\left(L^{p}, c_{0}\right)^{\#}\right)$ onto $\left(L^{p}, c_{0}\right)_{4}^{\sim}$.

We should note that

$$
\left(L^{p}, c_{0}\right)_{4}^{\sim} \subset\left(L^{p}, l^{\infty}\right)
$$

since

$$
\left(L^{p}, c_{0}\right) \subset\left(L^{p}, l^{\infty}\right)
$$

and by Theorem 5.1 this inclusion is proper because clearly constant functions belong to $\left(L^{p}, l^{\infty}\right)$ but they do not determine $c$-multipliers from $L^{1}$ to $\left(L^{p}, c_{0}\right)$.

For $\mu \in\left(L^{p}, l^{\infty}\right)$ we write $\mu^{0}$ to be the function on $G$ defined by

$$
\mu^{0}(x)=\int_{x+L} \mu(t) d t \quad(x \in G) .
$$

If $\left\{e_{n}\right\}$ is the a.i. in $L^{1}(G)$ formed by suitable multipliers of characteristic functions of compact neighborhoods of the identity, then the space $\left(L^{p}, c_{0}\right)$ is equal to

$$
\left\{\mu \in\left(L^{p}, l^{\infty}\right) \mid \mu^{0} \in\left(L^{p}, c_{0}\right)\right\}=\left\{\mu \in\left(L^{p}, l^{\infty}\right) \mid \mu * \chi_{U} \in\left(L^{p}, c_{0}\right)\right.
$$

for all (arbitrarily small) compact sets $U \subset G\}$.

6. Multipliers from amalgams and $M_{q}$ spaces to $L^{\infty}$ and $C_{0}$. We will start this section with the characterization of the space of $c$-multipliers from the algebras $\left(L^{p}, l^{1}\right)(1<p \leqq \infty),\left(C_{0}, l^{1}\right)$ to $L^{\infty}$.

THEOREM 6.1. Let $S$ be any of the algebras $\left(L^{p}, l^{1}\right)(1<p \leqq \mu),\left(C_{0}, l^{1}\right)$ and $T: S \rightarrow L^{\infty}$ be a linear operator. The following are equivalent:

i) $T \in c-M\left(S, L^{\infty}\right)$.

ii) There exists a unique $\mu \in S^{*}$ (if $\left.S=\left(L^{p}, l^{1}\right), 1<p<\infty\right), \mu \in M_{\infty}$ (otherwise) such that

$$
T f=\mu * f \text { for all } f \in S .
$$

iii) There exists a unique $\sigma \in S_{0}(\hat{G})^{*}(\sigma=\hat{\mu})$ such that

$$
(T f)^{\wedge}=\sigma \hat{f} \text { for all } f \in S \text {. }
$$

The correspondence between $T$ and $\mu$ establishes a continuous isomorphism from $c-M\left(S, L^{\infty}\right)$ onto $L^{\infty}$. The isomorphism is an isometry if

$$
S=\left(L^{p}, l^{1}\right) \quad(1<p<\infty) .
$$

Proof. Let $\left\{e_{n}\right\}$ be as in Section 5 and let $\mu$ be the element in $S_{0}(G)^{*}$ given by Theorem 3.2. As in the proof of Theorem 5.1, for $h \in S_{0}(G)$ 


$$
\begin{aligned}
|\langle h, \mu\rangle| & =\lim \left|\left\langle e_{n}, \mu * h\right\rangle\right|=\lim \left|\left\langle e_{n}, T h\right\rangle\right|=\lim \left|T h * e_{n}(0)\right| \\
& \leqq \lim \left\|T h * e_{n}\right\|_{\infty} \leqq\|T h\|_{\infty} \lim \left\|e_{n}\right\|_{1} \leqq\|T\|\|h\|_{S} .
\end{aligned}
$$

We conclude that $\mu \in S^{*}$ if

$$
S=\left(L^{p}, l^{1}\right)(1<p<\infty)
$$

and $\mu \in M_{\infty}$ if $S$ is either $\left(C_{0}, l^{1}\right)$ or $\left(L^{\infty}, l^{1}\right)$; and

$$
\|\mu\|_{p^{\prime} \infty} \leqq\|T\|
$$

by Proposition 2.6. The conclusion of the theorem follows from the Hölder inequality (Theorem 1.4).

The next theorem is an extension of Edwards' result for $L^{p}$ spaces [8, Theorem 3].

THEOREM 6.2. Let $B$ be any of the spaces $\left(L^{p}, l^{q}\right),\left(C_{0}, l^{q}\right),\left(L^{p}, c_{0}\right)$ $(1 \leqq p, q<\infty)$. If $T: B \rightarrow L^{\infty}$ is a linear operator, then the following are equivalent:

i) $T \in M\left(B, L^{\infty}\right)$.

ii) There exists a unique $\mu \in B^{*}$ such that

$$
T f=\mu * f \text { for all } f \in B \text {. }
$$

The correspondence between $T$ and $\mu$ establishes a continuous isomorphism from $M\left(B \cdot \mathrm{L}^{\infty}\right)$ onto $B^{*}$. The isomorphism is an isometry if

$$
B=\left(L^{p}, l^{q}\right) \text { or }\left(L^{p}, c_{0}\right) .
$$

Proof. We will prove the theorem for $B=\left(L^{p}, l^{q}\right)$. The remaining cases are similar (again remember that $\left(L^{1}, c_{0}\right)^{*}=\left(L^{\infty}, l^{1}\right)$ and note that $\left(C_{0}, l^{1}\right)$ is dense in $\left(C_{0}, l^{q}\right)$ and $\left.\left(L^{p}, c_{0}\right)\right)$.

If

$$
T \in M\left(\left(L^{p}, l^{q}\right), L^{\infty}\right),
$$

then $T \mid\left(L^{p}, l^{1}\right)$ belongs to $c-M\left(\left(L^{p}, l\right)^{1}, L^{\infty}\right)$ by Proposition 3.5 . So by Theorem 6.1 there exists a unique $\mu \in\left(L^{p^{\prime}}, l^{\infty}\right)$ such that

$$
T f=\mu * f \text { for all } f \in\left(L^{p}, l^{1}\right) .
$$

Since the map $f \rightarrow \mu * f$ from $\left(\left(L^{p}, l^{1}\right),\|\cdot\|_{p q}\right)$ into $L^{\infty}$ is continuous and $\left(L^{p}, l^{1}\right)$ is dense in $\left(L^{p}, l^{q}\right)([2$, Section 7, e) $]$ and $(1.1))$ we conclude that

$$
T f=\mu * f \quad \text { for all } f \in\left(L^{p}, l^{q}\right) .
$$

Similarly to the proof of Theorem 6.1 , for $h \in S_{0}(G)$ we have that

$$
|\langle h, \mu\rangle| \leqq\|T\|\|h\|_{p q}
$$

Again 


$$
\mu \in\left(L^{p^{\prime}}, l^{q^{\prime}}\right) \text { and }\|\mu\|_{p q} \leqq\|T\|
$$

by Proposition 2.6. The rest of the proof follows from Theorem 1.4.

Definition 6.4. For $1<p<\infty$, let $\left(L^{p^{\prime}}, l^{\infty}\right)_{1}^{\sim}$ be the space

$$
\left\{\mu \in\left(L^{p^{\prime}}, l^{\infty}\right) \mid\left\{\mu * e_{n}\right\} \subset C_{0}\right\}
$$

where $\left\{e_{n}\right\}$ is an a.i. in $\left(L^{p}, l^{1}\right)\left(\left(L^{p}, l^{1}\right)^{\#}(1<p<\infty)\right.$ is a Segal algebra [22, Theorem 4.16]).

THEOREM 6.5. Let $1<p<\infty$. If

$$
T:\left(L^{p}, l^{1}\right) \rightarrow C_{0}
$$

is a linear operator, then the following are equivalent:

i) $T \in c-M\left(\left(L^{p}, l^{1}\right), C_{0}\right)$.

ii) There exists a unique $\mu \in\left(L^{p}, l^{\infty}\right)_{1}^{\sim}$ such that

$$
T f=\mu * f \text { for all } f \in\left(L^{p}, l^{1}\right) \text {. }
$$

The correspondence between $T$ and $\mu$ establishes a continuous isomorphism from $c-M\left(\left(L^{p}, l^{1}\right), C_{0}\right)$ onto $\left(\left(L^{p}, l^{\infty}\right)_{1}^{\sim},\|\cdot\|_{p \infty}\right)$.

Proof. By Theorem 6.1 if i) holds then there exists a unique $\mu \in\left(L^{p^{\prime}}, l^{\infty}\right)$ such that

$$
T f=\mu * f \text { for all } f \in\left(L^{p}, l^{1}\right) .
$$

In particular

$$
\left\{T e_{n}=\mu * e_{n}\right\} \subset C_{0},
$$

hence

$$
\mu \in\left(L^{p^{\prime}}, l^{\infty}\right)_{1}^{\sim} .
$$

On the other hand if

$$
\mu \in\left(L^{p^{\prime}}, l^{\infty}\right)_{1}^{\sim} \text { and } f \in\left(L^{p}, l^{1}\right)
$$

then

$$
\left\{\mu * e_{n} * f\right\} \subset C_{0}
$$

because

$$
C_{0} *\left(L^{p}, l^{1}\right) \subset C_{0}
$$

(Theorem 1.6, i), see also (1.9) ) and

$$
\left\|\mu * e_{n} * f-\mu * f\right\|_{\infty} \leqq\|\mu\|_{p^{\prime} \infty}\left\|e_{n} * f-f\right\|_{p 1} .
$$

Since $\left\{e_{n}\right\}$ is an a.i. in $\left(L^{p}, l^{1}\right)$ we conclude that $\mu * f \in C_{0}$. Therefore $\mu$ determines a $c$-multiplier from $\left(L^{p}, l^{1}\right)$ to $C_{0}$. By the Hölder inequality 


$$
\|T\| \leqq\|\mu\|_{p^{\prime} \infty}
$$

and as in Theorem 6.1,

$$
\|\mu\|_{p^{\prime} \infty} \leqq\|T\| \text {. }
$$

Remark 6.6. Theorems 1.5, 6.1 and 6.2 imply that

i) $c-M\left(\left(C_{0}, l^{1}\right), L^{\infty}\right)=c-M\left(\left(C_{0}, l^{1}\right), C_{0}\right)$.

ii) $M\left(\left(L^{p}, l^{q}\right), L^{\infty}\right)=M\left(\left(L^{p}, l^{q}\right), C_{0}\right) \quad$ if $1 \leqq p<\infty, 1<q<\infty$.

iii) $M\left(\left(C_{0}, l^{q}\right), L^{\infty}\right)=M\left(\left(C_{0}, l^{q}\right), C_{0}\right) \quad$ if $1<q<\infty$.

iv) $M\left(\left(L^{p}, c_{0}\right), L^{\infty}\right)=M\left(\left(L^{p}, c_{0}\right), C_{0}\right) \quad$ if $1<p<\infty$.

v) $M\left(\left(L^{1}, c_{0}\right), L^{\infty}\right)=M\left(\left(L^{1}, c_{0}\right),\left(L^{\infty}, c_{0}\right)\right)$.

7. Inclusion results and the algebra $M_{1}(G)$.

Proposition 7.1. Let $S$ be any of the algebras $\left(L^{p}, l^{1}\right)(1 \leqq p \leqq \infty)$ or $\left(C_{0}, l^{1}\right)$ and $B$ be as in Proposition 3.5. Then

$$
c-M(S, B) \subset M(S, B) \text {. }
$$

Proof. If $T \in c-M(S, B)$ then for all $f \in S$,

$$
T f=\mu * f \text { for some } \mu \in S_{0}(G)^{*}
$$

(Theorem 3.2). Hence for $f \in S, h \in S_{0}(G)$, and $s \in G$,

$$
\begin{aligned}
\left\langle h, T f_{s}\right\rangle & =\left\langle h, \mu * f_{s}\right\rangle=\left\langle h * f_{s}, \mu\right\rangle=\left\langle h_{s} * f, \mu\right\rangle \\
& =\left\langle h_{s}, \mu * f\right\rangle=\left\langle h_{s}, T f\right\rangle=\left\langle h,(T f)_{s}\right\rangle .
\end{aligned}
$$

Since $B \in S_{0}(G)^{*}$ we conclude that $T$ commutes with translations.

COROLlary 7.2. Let $S$ be any of the algebras $\left(L^{p}, l^{1}\right)(1 \leqq p<\infty)$ $\left(L^{\infty}, l^{1}\right)^{w}$ or $\left(C_{0}, l^{1}\right)$ and $B$ be as in Theorem 3.2. Then

$$
c-M(S, B)=M(S, B) \text {. }
$$

We do not know if

$$
M\left(\left(L^{\infty}, l^{1}\right), A\right) \subset c-M\left(\left(L^{\infty} l^{1}\right), A\right)
$$

for some amalgam space or some $M_{q}$ space $A$.

Let $B$ be as in Theorem 3.2. If

$$
T: M_{1} \rightarrow B
$$

is a linear operator and $T$ has the form $T \mu=\varphi * \mu$ for some $\varphi \in B$ then by the properties of convolution and $M_{1}$-module [7] $T$ is a $c$-multiplier.

Conversely if $T$ is a $c$-multiplier from $M_{1}$ to $B$ and $\delta$ is the identity in $M_{1}$, then for $\mu \in M_{1}$ we have that

$$
T \mu=T(\delta * \mu)=T \delta * \mu=\varphi * \mu \quad \text { with } \varphi=T \delta .
$$


So we conclude that a linear operator $T: M_{1} \rightarrow B$ is a $c$-multiplier if and only if there exists a unique $\varphi \in B$ such that

$$
T \mu=\varphi * \mu \text { for all } \mu \in M_{1} \text {. }
$$

By the properties of convolution this implies that

$$
c-M\left(M_{1}, B\right) \subset M\left(M_{1}, B\right) \text {. }
$$

But we know that there exists a multiplier $T \in M\left(M_{1}, M_{1}\right)$ such that $T$ is not defined by the convolution with an element of $M_{1}[14$, p. 94]. So

$$
c-M\left(M_{1}, M_{q}\right) \neq M\left(M_{1}, M_{q}\right)(1 \leqq q \leqq \infty) .
$$

Indeed if

$$
c-M\left(M_{1}, M_{q}\right)=M\left(M_{1}, M_{q}\right) \text { and } T \in M\left(M_{1}, M_{1}\right)
$$

then $T \in M\left(M_{1}, M_{q}\right)$ since $M_{1} \subset M_{q}$, therefore

$$
\begin{aligned}
& T \in c-M\left(M_{1}, M_{q}\right) \text { and } \\
& T \mu=T \delta * \mu \text { for all } \mu \in M_{1} .
\end{aligned}
$$

This contradiction proves our claim.

We do not know if $M\left(M_{1}, A\right) \subset c-M\left(M_{1}, A\right)$ for some amalgam space $A$. However when we consider $M_{1}^{w}$ the situation is different. Since

$$
\delta * \mu_{s}=(\delta * \mu)_{s} \text { for all } \mu \in M_{1}
$$

and $s \in G$ we have by Proposition 3.5 and our previous discussion that

$$
M\left(M_{1}^{w}, B\right)=c-M\left(M_{1}^{w}, B\right)
$$

for $B$ as in Theorem 3.2.

Added in proof. The author learned recently that Theorems 5.1 and 6.2 were known to Professor H. Feichtinger some time ago although they were not published.

\section{REFERENCES}

1. J. P. Bertrandias and C. Dupuis, Transformation de Fourier su les espaces $l^{p}\left(L^{p^{\prime}}\right)$, Ann. Inst. Fourier, Grenoble 29 (1979), 189-206.

2. J. P. Bertrandias, C. Datry and C. Dupuis, Unions et intersections d'espaces $L^{p}$ invariantes par translation ou convolution, Ann. Inst. Fourier, Grenoble. 28 (1978), 53-84.

3. J. P. Bertrandias, Espaces $l^{p}(A)$ et $l^{\infty}(Q)$, (to appear).

4. J. T. Burnham and R. R. Goldberg, Multipliers from $L^{1}(G)$ to a Segal algebra, Bull. Inst. Math. Acad. Sinica. 2 (1974), 153-164.

5. R. C. Busby and H. A. Smith, Product-convolution operators and mixed-norm spaces, Trans. Am. Math. Soc. 263 (1981), 309-341.

6. M. Cowling, Some applications of Grothendieck's theory of topological tensor products in harmonic analysis, Math. Ann. 232 (1978), 273-285.

7. R. S. Doran and J. Wichmann, Approximate identities and factorization in Banach modules 768 (Springer-Verlag, 1970). 
8. R. E. Edwards, On factor functions, Pac. J. Math. 5 (1955), 367-378.

9. H. G. Feichtinger, Multipliers from $L^{1}(G)$ to a homogeneous Banach space, J. Math. Anal. Appl. 61 (1977), 341-356.

10. Un espace de Banach de distributions tempérées sur les groupes localement compact abeliens, C.R. Acad. Sci. Paris. 290 (1980), 791-794.

11. On a new Segal algebra, Mh. Math. 92 (1981), 269-289.

12. G. I. Gaudry, Quasimeasures and operators commuting with convolution, Pac. J. Math. 18 (1966), 461-476.

13. - Multipliers of type ( $p, q)$, Pac. J. Math. 18 (1966), 477-488.

14. - Topics in harmonic analysis, Lecture Notes (Department of Mathematics, Yale University, New Haven, Ct., 1969).

15. E. Hewitt and K. A. Ross, Abstract harmonic analysis I, II (Springer-Verlag, 1970, 1979).

16. F. Holland, Harmonic analysis on amalgams of $L^{p}$ and $L^{q}$, J. London Math. Soc. (2) 10 (1975), 295-305.

17. J. L. Kelly, I. Namioka and coauthors, Linear topological spaces (Princeton, N.J., D. van Nostrand, 1963).

18. T. Liu, A. van Rooij and J. Wang, On some group algebras of modules related to Wiener's algebra $M_{1}$, Pac. J. Math. 55 (1974), 507-520.

19. V. Losert, A characterization of the minimal strongly character invariant Segal algebra, Ann. Inst. Fourier, Grenoble 30 (1980), 129-139.

20. H. Reiter, $L^{1}$-algebras and Segal algebras, 231 (Springer-Verlag, 1971).

21. J. Stewart, Fourier transforms of unbounded measures, Can. J. Math. 31 (1979), 1281-1292.

22. M. L. Torres de Squire, Amalgams of $L^{p}$ and $l^{q}$, Ph. D. Thesis, McMaster University (1984).

University of Regina,

Regina, Saskatchewan 